\title{
Keeping an Eye on the Truth? Pupil size changes associated with recognition memory
}

\author{
Becky Heaver and Sam B Hutton* \\ School of Psychology, University of Sussex, Brighton, UK
}

\begin{abstract}
During recognition memory tests participants' pupils dilate more when they view old items compared to novel items. We sought to replicate this "pupil old/new effect" and to determine its relationship to participants' responses. We compared changes in pupil size during recognition when participants were given standard recognition memory instructions, instructions to feign amnesia and instructions to report all items as new. Participants' pupils dilated more to old items compared to new items under all three instruction conditions. This finding suggests that the increase in pupil size that occurs when participants encounter previously studied items is not under conscious control. Given that pupil size can be reliably and simply measured, the pupil old/new effect may have potential in clinical settings as a means for determining whether patients are feigning memory loss.
\end{abstract}

Keywords: Recognition memory; Malingering; Pupil size; Deception.

Since the 1960s researchers have explored the relationship between pupil size and cognitive function (see Janisse, 1977, and Beatty \& LuceroWagoner, 2000, for reviews). Much of the early research focussed on the relationship between pupil size and concepts such as "arousal" or "mental effort” (eg Hess \& Polt, 1964; Beatty \& Kahneman, 1966; see Kahneman, 1973, and Janisse, 1977, for reviews), but some early studies documented a relationship between pupil size and mnemonic processes (e.g. Bradshaw, 1967; Bradshaw, 1968; Gardner, Mo, \& Borrego, 1974; Gardner, Mo, \& Krinsky, 1974; Garrett, Harrison, \& Kelly, 1989). For example, Gardner, Mo and Borrego (1974) presented participants with a series of nonsense words. Some of the items had been presented to participants prior to the recognition test (familiar nonwords) whereas other items were unfamiliar. They report that participants' pupils dilated on presentation of the familiar nonwords but constricted when unfamiliar nonwords were presented. The same effect was observed in a similar study using consonant trigrams (Gardner, Mo, \& Krinsky, 1974b). More recently a study by Maw and Pomplun (2004) found that famous faces produced a brief increase in pupil size relative to non-famous faces, and these authors proposed that the pupil response reflected some kind of recognition processes. They did not explore whether non-famous familiar faces produced the same effect, or whether it generalised to other types of stimulus.

Building on this research, Otero, Weekes and Hutton (2006), reported that the pupil dilation effect could also be observed during standard recognition memory test, such that stimuli previously encountered during the study phase were associated with greater pupil diameter increase than unfamiliar new stimuli. Võ et al. (2008) also reported a "pupil old/new effect" using a recognition memory task comprising words that varied in emotional content. They asked participants to rapidly assess whether items were old or new, and found that hits were 
associated with a larger pupil size than correct rejections, and that recognition was subject to an emotion-activated bias whereby the pupil old/new effect was attenuated by words with positive or negative emotional valence.

Similar "old/new effects" have been extensively documented in the ERP literature. For example earlier and larger parietal late positive components (LPCs) have been found in response to the presentation of old learned items compared to new items during recognition memory (Warren, 1980; van Hooff, Brunia, \& Allen, 1996; Friedman \& Johnson, 2000). One potential role for psychophysiological indices of mnemonic processes such as the ERP and pupil old/new effects is in identifying people who feign memory loss (eg Browndyke et al., 2008; Tardif, Barry, Fox, \& Johnstone, 2000; van Hooff, Sargeant, Foster, \& Schmand, 2009). Tardif et al. (2000) for example reasoned that if the ERP old/new effect is not under conscious control then it should be detectable in people feigning amnesia. In their experiment, participants learned a set of words before completing a recognition memory test. Half the participants were given standard test instructions to respond old to the learned words and new to new words. The other half were asked to perform deliberately poorly on the test. The ERP measures showed no difference in the magnitude and topography of the old/new ERP effect between the groups, suggesting that the malingering group did in fact have intact recognition of the learned words.

Given the comparative ease with which pupil measurements can be made, it is important to establish whether a similar approach might be feasible using the pupil old/new effect. Some early evidence suggests that, like the ERP old/new effect, the pupil old/new effect is not under voluntary control. Clark and Johnson (1970) informed participants that their pupil would increase or decrease in size during a short term memory task, or, in a control condition, did not mention pupil size at all. They found that pupil size increased to a similar extent in each condition.

Here we set out to replicate the pupil old/new effect and to investigate its relationship to participants' responses. If, like the ERP old/new effect, the pupil old/new effect is not under voluntary control, pupil size should increase for old items compared to new items even when participants feign amnesia and pretend not to recognise learned stimuli in the 'malingering' condition. As pupil size has been shown to increase in relation to cognitive load (e.g. Khaneman \& Beatty, 1966; Porter, Trosciancko \& Gilchrist, 2007) and it is generally assumed that for most participants deception involves more cognitive effort than telling the truth, we included a third "single response" condition, in which participants answered "new" to all items. We predicted that pupil size would also increase for old items compared to new items in this condition.

\section{Method}

\section{Participants}

Twenty-six participants (6 male; age range: $19-30, M$ $=23.1, S D=3.3$ ), with normal or corrected-to-normal vision in at least one eye were recruited from the student psychology participation pool at the University of Sussex, and through personal contact. Participants were briefed with a detailed consent form and verbal description, and invited to ask questions. Written consent was obtained prior to testing and participants were fully debriefed. The experiment was approved by the relevant ethics committee.

\section{Materials}

Three study lists were created for the learning phase, each list comprising 40 nouns selected from the MRC Psycholinguistic Database. For the recognition test, three lists were constructed, each containing the 40 items that were on the corresponding study list and 40 new nouns that were not. All items were seven letters long, matched for familiarity and imageability, according to the K-F norms (familiarity range $=301-646, M=493.62$, imageability range $=261-630, M=497.08)$. The three parallel sets of study lists and recognition tests formed blocks $A$ to $C$, and were presented in black 20-pt Mono-spaced font on a light grey background on a 21 inch CRT monitor. Participants viewed the monitor from a distance of $70 \mathrm{~cm}$ and the visual angle subtended by the words was approximately $3^{\circ}$. Eye movements were recorded with an Eyelink II (SR-Research, Ontario), with a sampling rate of $500 \mathrm{~Hz}$. 


\section{Design and Procedure}

At the start of the experiment, participants were asked to imagine that they had recently been involved in a car accident and as a result were unconscious for 15 minutes and had to spend one night in hospital for observation. They were told that their condition had gradually improved over the following months and they had now made a full recovery. They were asked to imagine that the purpose of the test that they were are about to undertake was to determine whether the accident has produced any long-term memory impairments due to brain damage. This scenario was adapted from van Hooff et al. (2009).

In a within-participant design each participant completed three separate recognition memory tests. Each test contained a learning phase and a recognition phase. During the learning phase, 40 study list target items were presented on screen for 2000 ms with $1000 \mathrm{~ms}$ between words, and participants were asked to remember the items. During the testing phase, 80 recognition list items (40 old targets and 40 new distracters) were presented for $1750 \mathrm{~ms}$, each following a $250 \mathrm{~ms}$ mask ("\&\&\&\&\&\&"). The mask reappeared after 2000 ms and participant stated whether the word was old (previously encountered in the learning phase) or new (not previously encountered). Participants were then presented with a screen prompting them to estimate their confidence in their decision with a number between 1 and 5 , where 1 represented a complete guess and 5 represented total confidence. This screen was then replaced by a drift-correction dot in the centre of the screen in preparation for the start of the next trial.

In the standard instructions condition participants were asked to perform as accurately as possible during the recognition test. In the malingering condition, participants were asked to produce responses that would convince an examiner that they still had a memory impairment. They were advised their responses should be presented in a "believable" manner, and major exaggerations, such as not remembering anything, should be avoided. To simulate real-world compensation participants were told that $£ 10$-worth of book vouchers would be awarded to the individual who best managed to simulate a believable memory deficit. In a "single response" control condition, participants were instructed to simply answer "new" to all items, regardless of whether they knew them to be old or new. This condition was intended to mimic a simple strategy that might be used by people feigning amnesia, and also allowed us to rule out any potential confounding influences on pupil size that might result from the increased cognitive effort required to generate incorrect responses in the malingering condition.

In order to control against list and order effects, the condition order was rotated across participants. In order to determine whether any effects on pupil size differed as a function of condition order, this variable was added as a factor to all initial statistical analyses. There were no main effects of order nor did it interact with any other factors, so for ease of interpretation, it is not included in the results section. In order to prevent the recognition phase instructions influencing behaviour during the learning phase (ie participants may not have concentrated on the study items if they knew they were going to be saying "new" to all items), instructions for the recognition phase were provided after the learning phase in each condition.

\section{Pupil recording}

Maximum pupil size was recorded from the right eye during the time the item was on screen during the recognition test. Due to constant fluctuation in pupil size over time, and variation between individuals, a pupil dilation ratio (PDR) was calculated. The PDR was calculated by dividing the maximum pupil size during the $1750 \mathrm{~ms}$ the word was present by the maximum pupil size during the $250 \mathrm{~ms}$ baseline period prior to stimulus presentation when the stimulus mask was on screen.

\section{Results}

\section{Behavioural Data}

The proportion of old responses to old and new items was calculated for standard and malingering conditions (no old responses were made in the single response control condition). A 2 (item type: old vs new) by 2 (condition: standard vs malingering) repeated measures ANOVA showed a significant main effect of item type - in general participants 
responded old more often to old items than new items $\left(F(1,25)=162.5, M S E=0.027, p<.001, \eta_{p}{ }^{2}=\right.$ .87), a significant main effect of condition - in general participants responded old more often in the standard condition $(F(1,25)=4.16, M S E=0.012, p=$ $\left..05, \eta_{p}^{2}=.14\right)$ and a significant interaction between item type and condition $(F(1,25)=30.95, M S E=$ $\left.0.03, p<.001, \eta_{p}^{2}=.55\right)$. The interaction occurs because participants responded old to old items significantly more in the standard condition $(M=$ $0.79, S D=0.12)$ than in the malingering condition $(M$ $=0.56, S D=0.15 ; t(25)=5.55, p<.001)$, whereas participants responded old to new items significantly more in the malingering condition $(M=0.34, S D=$ $0.15)$ than in the standard condition $(M=0.20, S D=$ $0.15 ; t(25)=3.79, p=.001$; see Figure 1$)$.

Confidence ratings were analysed with a 3 (condition: standard, malingering, single response) by 2 (item type: old vs new) repeated measures ANOVA. The main effect of item type was significant $\left(F(1,25)=27.06, M S E=0.062, p<.001, \eta_{p}{ }^{2}=.52\right)$ with average confidence levels for old words (3.33) higher than for new words (2.96). The main effect of condition was significant $(F(2,50)=69.58, M S E=$ $\left.0.523, p<.001, \eta_{p}{ }^{2}=.74\right)$ with average confidence levels close to ceiling in the single response condition (4.84) and lowest in the malingering condition (3.17). Average confidence in the standard condition was 3.90. A significant condition by item type interaction $(F(2,50)=15.64, M S E=0.058, p<$ $.001, \eta_{p}^{2}=.39$ ) arose because confidence ratings were significantly higher for old compared to new items in the standard condition ( 4.15 vs $3.70, t(25)=$ $7.25, p<.001)$ and malingering condition $(3.26$ vs $3.09, t(25)=2.58, p<.02)$ but not in the single response condition (4.82 vs $4.86, n s)$.

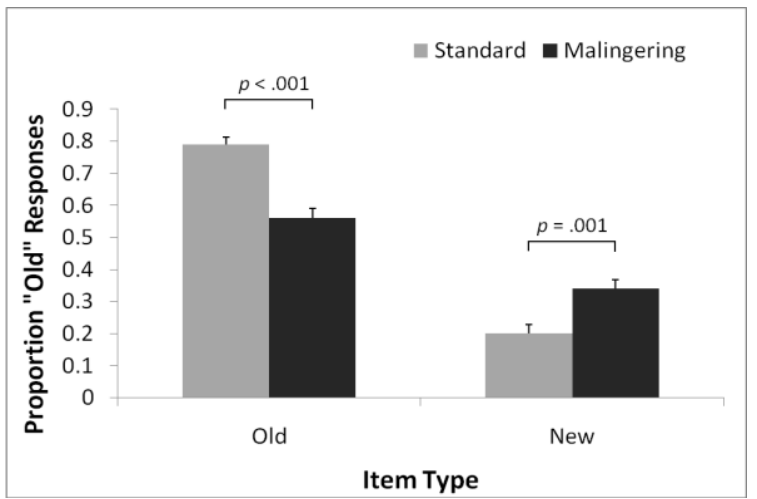

Figure 1: Proportion of old responses to old and new items for standard and malingering conditions. Error bars are standard error of the mean.

\section{Pupil Size Data}

Average PDR for old and new words in the three conditions were compared with a $2 \times 3$ ANOVA with item type (old vs new) and condition (standard vs malingering vs single response) as within subject factors. There was a main effect of item type $\left(F(1,25)=47.02, M S E<0.001, p<.001, \eta_{p}^{2}=.65\right)-$ the PDR was larger for old items compared to new items regardless of whether people were instructed to respond veridically, feign amnesia or identify all items as new. The main effect of condition was also significant $(F(2,50)=24.37, M S E=0.001, p=.01$, $\eta_{p}^{2}=.49$ ). Average PDRs to old and new items were higher in the standard condition compared to the malingering condition and higher again in the malingering condition compared to the single response condition. These differences were significant for both old and new items (all ts $>2.6, p s$ $<.05)$. These main effects were, however, qualified by a significant item type by condition interaction $\left(F(1.96,48.89)=5.17, M S E<0.001, p=.01, \eta_{p}^{2}=\right.$ .17; dfs are Greenhouse-Geisser adjusted due to violations of sphericity). The interaction arises because the average increase in pupil size is smaller in the single response condition $(M=0.009, S D=$ $0.017)$ than in the standard $(M=0.025, S D=0.021$, $t(25)=3.34, p=.003, r=.31)$ or malingering conditions $(M=0.018, S D=0.021, t(25)=2.07, p<$ $.05, r=.15)$ (see Figure 2).

As participant response (old vs new) was not meaningful in the malingering condition, it was not included as a factor in the analysis above. However, it is important to establish whether, in the standard condition, pupil size increases for old items that are not correctly recognised (misses), as a patient with

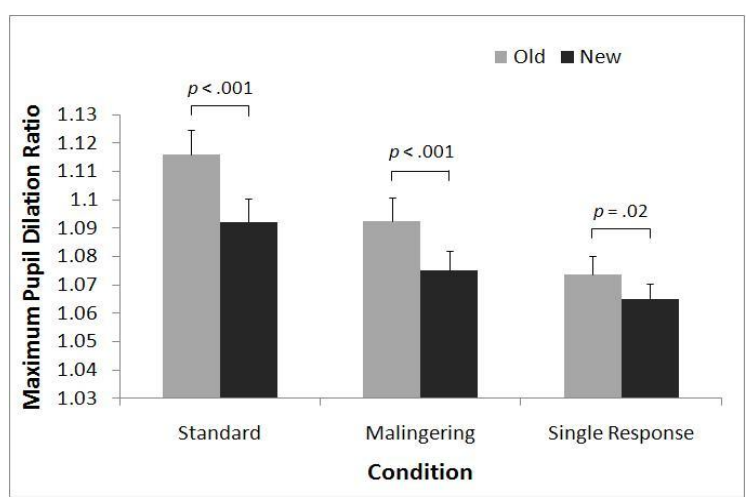

Figure 2: Maximum Pupil Dilation Ratio for old and new items in standard, malingering and single response conditions. Error bars are standard error of the mean. 
genuine memory problems might show poor explicit recognition memory but an increase in pupil size when targets are presented. Four participants made fewer than 5 misses, and were therefore excluded from this analysis. Average PDR to missed old items was 1.09 - the same PDR as was observed for correct rejections $(t(20)=.02$, ns). PDR is a function of baseline pupil size, so we therefore compared baseline pupil sizes in the three conditions to ensure that any differences in PDR were not due to baseline differences. The difference was not significant $(F(1.63,40.76)=1.90, p=.17$, ns; $d f$ are Greenhouse-Geisser adjusted due to violations of sphericity).

Participants made higher confidence ratings on average to their correct "old" judgments compared to their correct "new" judgements in the standard and malingering conditions. It is important to establish the extent to which the increase in PDR that occurs when participants view old items is associated with the increase in confidence that is associated with giving an old compared to new response. PDR was significantly higher in the standard condition for high confidence (4 or $5 ; M=1.13, S D=0.055$ ) compared to low confidence $(<4 ; M=1.10, S D=0.048)$ correct old judgements $(t(15)=3.41, p=.004, r=$ $.44)$. This analysis was restricted to the 16 participants who had at least 5 high and low confidence correct old judgements, and to the standard condition because confidence judgements were not meaningful in the malingering condition (it is impossible to determine whether reduced confidence reflects a genuine uncertainty as to the correctness of their response or an understandable attempt by participants to give the impression that they have a poor memory).

Despite being overall slightly less confident in their correct rejections than their correct recognitions, participants made significant numbers of high confidence correct rejections. In order to further explore the relationship between confidence and PDR we compared PDR for correctly identified old and new items to which participants gave high confidence responses. PDR to old items that were correctly identified with a high degree of confidence was greater than the PDR to new items that were correctly identified with high confidence for all three conditions (standard: $t(24)=5.43, p<.001, r=.55$; malingering: $t(23)=4.03, p=.001, r=.41$; single response: $t(25)=2.14, p=.04, r=.15)$.

\section{Discussion}

We sought to replicate the pupil old/new effect and determine its relationship with participant's responses. The size of participants' pupils increased to a greater extent when they viewed old items compared to novel items in a standard recognition test and, critically, this effect was also observed when participants were instructed to feign amnesia, or even just give a "new" response to all items.

The finding that under standard recognition memory instructions, participants' relative increase in pupil size is greater when they view old items compared to new items replicates previous research (e.g. Bradshaw, 1967; Bradshaw, 1968; Gardner, Mo, \& Borrego, 1974, Gardner, Mo, \& Krinksky, 1974; Garrett et al., 1989; Maw \& Pomplun, 2004; Otero et al, 2006, 2011; Võ et al., 2008) and demonstrates that the "pupil old/new effect" is a robust phenomenon. It has been suggested that the pupil old/new effect reflects cognitively demanding recollective processes that occur during the recognition of old items but not the correct rejection of new items (Võ et al., 2008). This interpretation builds on an extensive body of work demonstrating that increases in pupil size occur as processing demands or cognitive load increase (see e.g. Kahneman, 1973). However, it is not clear why recognition of previously presented items should necessarily be more cognitively demanding than the correct rejection of novel items - for example correct rejection may involve an effortful memory search, and studies have found that it typically takes longer than correct recognition (e.g. Ratcliff \& Murdock, 1976).

Our finding that the pupil old/new effect was also observed in a "single response" condition (in which participants simply had to respond "new" to all items) may also seem problematic for an interpretation of the pupil old/new effect based on cognitive effort - it could be argued that it takes the same amount of cognitive effort to respond "new" to a word during a recognition test when that word is old as it does when the word is new. It is possible, however, (and, as we argue below, likely) that despite the lack of any requirement for a genuine old/new decision to be made in the single response condition, recognition (and accompanying mnemonic processes) still occurred when people encountered old items. If it is assumed that it is these mnemonic 
processes themselves (as opposed to the cognitive effort they may involve) that are associated with the increase in pupil size, then the present pattern of results would be expected. The pupil old/new effect was greatest when participants were given standard instructions to make a genuine old/new decision for each word and diminished somewhat in the malingering and single response conditions. In the absence of any requirement to respond accurately in the malingering condition participants may have "preloaded" either an old or new response. This preloading strategy was required in the single response condition. As a result in both malingering conditions less genuine recognition / recollection may have occurred, with a resulting reduction in the magnitude of the overall pupil old/new effect when averaged across trials.

Another possibility is that the increase in pupil size that occurs when participants view old items during a recognition test somehow reflects differences in confidence associated with correct recognition of old items compared to the correct rejection of new items. Participants did indeed give higher confidence ratings on average to their correct "old" judgments than their correct "new" judgements in both the standard and malingering conditions. If (as suggested above) the pupil old/new effect reflects the operation of mnemonic processes during recognition then the extent of the pupil size increase might be expected to be associated with confidence. Recent models of recognition memory have moved away from the idea that recollection is an 'all or nothing' process, instead suggesting that, like familiarity, the recollection signal may vary along a continuum. If the aggregate "strength of memory" signal exceeds a certain threshold the item is identified as old (Wixted \& Stretch, 2004; Wixted, 2007). If confidence ratings are taken as a reflection of participant's subjective experience of the strength of this aggregate signal, and the pupil size increase reflects the cognitive processes that drive this signal, then pupil size increases should be greater for high compared to low confidence judgments, as was indeed the case.

Despite this relationship, the pupil old / new effect does not simply reflect the difference in confidence between correct recognition of targets and the correct rejection of distracters. Participants can, of course, be highly confident that an item was not on the study list. When PDR was compared between only the highly confident (rated 4 or 5) correctly identified old and new items, the old/new effect remained significant in all conditions. Similarly, participants were significantly more confident when making correct rejections than false alarms, but there were no differences in pupil size. These findings suggest that whilst confidence may be related to the magnitude of the pupil old/new effect, the increase in pupil size that occurs when participants view old items does not simply reflect a "confidence signal".

The key finding of the present study is the demonstration that the pupil old/new effect occurs even when participants are deliberately giving wrong answers under instructions to malinger, and when they are instructed to simply identify all items as new. These results support Clark and Johnson (1970)'s finding that the pupil effect is not under voluntary control and show that it is independent of participants' actual response. A similar argument has been made concerning the ERP old/new effect, and has been used to support its potential use as an index of malingering (Tardif et al., 2000; van Hooff et al., 2009). In a recent study, however, the ERP old/new effect was not observed in a group of participants instructed to malinger (Vagnini, Berry, Clark, \& Jiang, 2008). Differences in procedure, in particular whether participants were asked to feign amnesia before or after learning the word list, may account for the different findings. We are currently conducting experiments exploring the relationship between the pupil old/new effect and the ERP old/new effect.

The finding that the pupil old/new effect can be reliably detected even when participants are feigning amnesia and are reporting that they believe the items to be new, might have implications for individuals and organisations who administer neuropsychological recognition memory tests in clinical or forensic settings. The absence of a significant difference in PDR between old items missed and correct rejections of new items in the standard condition suggests that if a patient with legitimate memory problems makes a genuine miss we they would not be incorrectly identified as a malingerer on the basis of their pupil size. Clearly it will be important to establish how pupil size changes in genuinely memory-impaired populations when they perform a recognition memory test. Laeng et al. (2007) recently investigated the pupil old/new effect 
in three patients with amnesia resulting from hippocampal lesions. They found that a larger pupil response occurs for new words compared to old words in these patients. If a similar pattern occurs in other memory impaired populations, or even if they show no difference in pupil size between old and new items, the increase observed in our malingering sample would serve as a useful indication that their recognition memory is in fact intact. It will also be important to establish whether the pupil old/new effect can be diminished by countermeasures, such as participants deliberately performing an effortful cognitive task when responding to new items, in order to malinger.

In conclusion, this study confirms and extends previous research demonstrating that pupil size increases more for previously encountered stimuli than for new items during a recognition memory test. Critically, this increase appears to be independent of behavioural responses and may have potential as a comparatively simple and easy tool with which to detect patients feigning amnesia.

\section{References}

Beatty, J., \& Kahneman, D. (1966). Pupillary changes in two memory tasks. Psychonomic Science, 5, 371-372.

Beatty, J., \& Lucero-Wagoner, B. (2000). The pupillary system. In J. T. Cacioppo, L. G. Tassinary \& G. G. Berntson (Eds.), Handbook of psychophysiology (2nd ed., pp. 142-162). United States of America: Cambridge University Press.

Bradshaw, J. (1967). Pupil size as a measure of arousal during information processing. Nature, 216(5114), 515516.

Bradshaw, J. L. (1968). Pupil size and problem solving. Quarterly Journal of Experimental Psychology, 20, 116122.

Browndyke, J. N., Paskavitz, J., Sweet, L. H., Cohen, R. A., Tucker, K. A., Welsh-Bohmer, K. A., Burkey, J. R., \& Schmechel, D. E. (2008). Neuroanatomical correlates of malingered memory impairment: Event-related fMRI of deception on a recognition memory task. Brain Injury, 22(6), 481-489.

Clark, W. R., \& Johnson, D. A. (1970). Effects of instructional set on pupillary responses during a shortterm memory task. Journal of Experimental Psychology, 85(2), 315-317.

Friedman, D. \& Johnson Jr., R. (2000). Event-related potential (ERP) studies of memory encoding and retrieval: a selective review. Microscopy Research and Technique, 51, 6-28.
Gardner, R. M., Mo, S. S., \& Borrego, R. (1974). Inhibition of pupillary orienting reflex by novelty in conjunction with recognition memory. Bulletin of the Psychonomic Society, 3(3-B), 237-238.

Gardner, R. M., Mo, S. S., \& Krinsky, R. (1974). Inhibition of pupillary orienting reflex by heteromodal novelty. Bulletin of the Psychonomic Society, 4(5-B), 510-512.

Garrett, J. C., Harrison, D. W., \& Kelly, P. L. (1989). Pupillometric assessment of arousal to sexual stimuli Novelty effects or preference. Archives of Sexual Behavior, 18(3), 191-201.

Hess, E. H., \& Polt, J. M. (1964). Pupil size in relation to mental activity in simple problem solving. Science, 143, 1190-1192.

Janisse, M. P. (1977). Pupillometry: The psychology of the pupillary response. Washington, D. C.: Hemisphere Publishing Corporation.

Kahneman, D. (1973). Attention and effort. New Jersey, USA: Prentice Hall.

Kahneman, D., \& Beatty, J. (1966). Pupil diameter and load on memory. Science, 154, 1583-1585.

Laeng, B., Waterloo, K., Johnsen, S. H., Bakke, S. J., Låg, T., Simonsen, S. S., \& Høgsæt, J. (2007). The eyes remember it: Oculography and pupillometry during recollection in three amnesic patients. Journal of Cognitive Neuroscience, 19(11), 1888-1904.

Maw, N. \& Pomplun, M. (2004). Studying human face recognition with the gaze-contingent window technique. In K. Forbus, D. Gentner \& T. Regier (Eds.), Proceedings of the Twenty-Sixth Annual Meeting of the Cognitive Science Society, 2004, Chicago, Illinois.

Otero, S., Weeks, B., \& Hutton, S. (2006). A novel association between pupil size and recollective experience during recognition memory. Proceedings of the Second Biennial Conference on Cognitive Science, St Petersburg, Russia.

Otero, S. C., Weeks, B. S., \& Hutton, S. B. (2011). Pupil size changes during recognition memory. Psychophysiology, 48. Doi: 10.1111/j.1469-8986.2011.01217.x

Porter, G., Troscianko, T., \& Gilchrist, I. D. (2007). Effort during visual search and counting: Insights from pupillometry. The Quarterly Journal of Experimental Psychology, 60, 211-229.

Ratcliff, R., \& Murdock, B. B., Jr. (1976). Retrieval processes in recognition memory. Psychological Review, 83, 190-214.

Tardif, H. P., Barry, R. J., Fox, A. M., \& Johnstone, S. J. (2000). Detection of feigned recognition memory impairment using the old/new effect of the event-related potential. International Journal of Psychophysiology, 36, 1-9.

Vagnini, V. L., Berry, D. T. R., Clark, J. A., Jiang, Y. (2008). New measures to detect malingered neurocognitive deficit: Applying reaction time and event-related potentials. Journal of Clinical and Experimental Neuropsychology, 30(7), 766-776. 
van Hooff, J. C., Brunia, C. H. M., \& Allen, J. J. B. (1996). Event-related potentials as indirect measures memory. International Journal of Psychophysiology, 21, 15-31.

van Hooff, J.C., Sargeant, E., Foster, J.K., \& Schmand, B.A. (2009). Identifying deliberate attempts to fake memory impairment through the combined use of reaction time and event-related potential measures. International Journal of Psychophysiology, 73, 246-256.

Võ, M. L. H., Jacobs, A. M., Kuchinke, L., Hofmann, M., Conrad, M., Schacht, A., \& Hutzler, F. (2008). The coupling of emotion and cognition in the eye:
Introducing the pupil old/new effect. Psychophysiology, 45(1), 130-140.

Warren, L. R. (1980). Evoked-potential correlates of recognition memory. Biological Psychology, 11, 21-35.

Wixted, J. T. (2007). Dual-process theory and signaldetection theory of recognition memory. Psychological Review, 114, 152-176.

Wixted, J. T. \& Stretch, V. (2004). In defense of the signaldetection interpretation of Remember/Know judgments. Psychonomic Bulletin \& Review, 11, 616-641. 